\title{
Performance of Laser Fluorescence for Residual Caries Detection in Primary Teeth
}

\author{
Taskin Gurbuz \\ Yucel Yilmaz \\ Fatih Sengulc
}

\section{ABSTRACT}

Objectives: The aim of this study was to evaluate the performance of a visual-tactile examination and a laser fluorescence device for detecting residual dentinal caries after carious dentin removal with bur excavation, hand excavation and chemomechanical excavation [Carisolv ${ }^{\mathrm{TM}}$ ].

Methods: Thirty extracted coronal caries primary second molars were used. The caries infected dentin has been removed. A blinded examiner checked all cavities for residual caries using a visualtactile examination and laser fluorescence. Then the teeth were sectioned through the prepared cavities and the two halves of each tooth were processed for light microscopy and Scanning Electron Microscopy (SEM). The presence or absence of residual caries was verified using polarized light microscopy as the gold standard. The cavity floor dentin after removing carious dentin was examined using SEM.

Results: In among all groups a significant difference is determined between visual-tactile examination and laser fluorescence $(P<.05)$. There was an agreement between laser fluorescence or visual-tactile examination and histological gold standard ( $P>$.05). In addition, SEM images of the surfaces with the caries removed have shown that a vast majority of the tubule openings is observed to be open in the samples of the Carisolv group in opposition to the other groups.

Conclusions: The laser fluorescence system could be effective in checking the removal by other methods, such as bur or Carisolv and avoid excessive removal of the sound dentin. (Eur J Dent 2008;2:176-184]

Key words: Caries removal techniques; Carisolv; Laser fluorescence; Residual caries; Carious dentin; Children.

a Assistant Professor, Department of Pedodontics, Faculty Dentistry, Ataturk University, Erzurum, Turkey.

b Associate Professor, Department of Pedodontics, Faculty Dentistry, Ataturk University, Erzurum, Turkey.

Research Assistant, Department of Pedodontics,

Faculty Dentistry, Ataturk University, Erzurum, Turkey.

- Corresponding author: Taskin Gurbuz Atatürk Universitesi, Dis Hekimligi Fakultesi,

Pedodonti Ana Bilim Dali, Erzurum, Turkey.

Phone : +904422311777

Fax : $\quad$ +90 (442) 2360945

E-mail : gurbuzt25dyahoo.com

\section{INTRODUCTION}

In the treatment of carious dentin, a number of techniqueshavebeenused to date. Recentoutcomes obtained from experimental studies about tooth caries pathology, natural protection mechanism of teeth and advances in dental restorative materials have resulted in the necessity of the removal of only infected carious dentin. ${ }^{1}$ The hardness and, to a lesser extent, the color of dentin are presently the main parameters used by dental practitioners 
to differentiate between infected and non-infected dentin during caries excavation. ${ }^{1}$ Histologically, it is possible to distinguish between an outer unremineralizable and an inner remineralizable layer of carious tissue. ${ }^{2}$

Researchers have stated that in the determination of the complete removal of the carious dentin, color (visual criteria) and hardness (tactile criteria) have been used as criteria for the clinical assessment. ${ }^{3}$ Estimation of hardness of the remaining dentin by tactile procedures, however, may not be a reliable guide for the clinical removal of caries. ${ }^{3}$ Discoloration is considered reliable only in chronic caries where it is usually marked, and that the extent of bacterial invasion follows closely the discoloration front. In acute caries, the zones of discoloration are less evident, and bacterial invasion is usually diffuse and extends beyond the discoloration front. ${ }^{3}$ Thus, discoloration is not a reliable guide for the removal of infected dentin.

Caries detecting dyes have been used to facilitate clinical discrimination of carious dentin from sound dentin during caries removal. ${ }^{4}$ The use of these dyes, however, does not provide a completely objective method for assessment of caries removal. Excessive removal of dentin has been reported when these dyes were used to remove caries. ${ }^{5}$

The laser fluorescence device, DIAGNOdent (KaVo Co., Biberach, Germany), has been developed for objective caries diagnosis. ${ }^{6}$ Also, DIAGNOdent was shown to be applicable for caries diagnosis during caries removal. ${ }^{7}$ With this device, the tooth surface is illuminated with pulses of red laser light, and the fluorescence emitted from the surface is analyzed and quantified. Caries process alters the amount of fluorescence, which can be seen as an elevated reading. The fluorescence emitted from a test surface is displayed as numerical values ranging from 0 to 99 , with deeper carious lesions producing higher values. ${ }^{7,8}$ DIAGNOdent exhibited greater sensitivity than caries detecting dye in caries detection. ${ }^{9}$ Thus, apart from the use of caries detecting dyes, the DIAGNOdent may be used to evaluate the extent of demineralization of dentin during caries removal. ${ }^{10}$ However, further detailed studies are needed before DIAGNOdent values can be used to differentiate the layers of carious dentin.

Studies carried out so far show that the layer of the carious dentine, which is rich in bacteria, unremineralizable and has necrotic tissue remaining on its surface, should be removed by any technique. ${ }^{1,3,5}$ However, secondary or reparative dentine developed as a positive response under the infected region should not be removed. ${ }^{5}$ Currently, there is a growing interest to develop minimal invasive techniques such as chemomechanical caries removal in the treatment of the carious lesion. The chemomechanical method does not produce an uncomfortable machine sound as do rotary cutting instruments, and tooth substance can be removed without pain. ${ }^{11}$ Both clinical reliability and accepting of patient as well as activity of the carious removal have a great interest in pediatric dentistry. ${ }^{11}$ Therefore, in recent years, the use of the chemomechanical method for treating dental caries has become widespread. However, Splieth et $\mathrm{al}^{12}$ reported that more than $50 \mu \mathrm{m}$ of carious dentin were left following Carisolv treatment when compared to the conventional mechanical tooth preparation. Yazici et al ${ }^{13}$ showed that the residual bacteria mainly at the dentinoenamel junction following caries removal with Carisolv. The firm feeling of sound dentin is not always differentiated, and color and sound do not give a true indication of sound dentin. ${ }^{13}$ Hossain et $\mathrm{al}^{14}$ reported that Carisolv is capable of removing complete carious dentin if proper clinical guide is applied.

The laser fluorescence score depends on the amount of metabolic by-products of cariescausing bacteria and fluorescent protoporphyrin present, $^{15}$ and the color of carious dentin. ${ }^{7}$ Moreover, differences in the structure of dentine surface being evaluated influence the results using the DIAGNOdent. Thus, in order to use the DIAGNOdent for removal of caries, it is necessary to clarify the influence that the evaluated dentin's structure (for example, the existence of caries or the course of dentinal tubules or the presence of a smear layer) has on the DIAGNOdent readings.

The aim of this in vitro study was to compare the performance of a visual-tactile examination and a laser fluorescence device (DIAGNOdent) for detection of residual dentinal caries after carious dentin removal with the bur excavation, hand excavation and Carisolv system. Also, we also assessed the surface morphologies using scanning electron microscope (SEM). The hypothesis tested was that not to be of influence of the structures of 
the prepared dentin surfaces after different caries removal methods on the results of diagnosis using the DIAGNOdent.

\section{MATERIALS AND METHODS}

The protocol of this investigation was approved by the Ethics Committee of the Faculty of Dentistry, Ataturk University. In this study, a total of thirty freshly extracted for orthodontic reasons and exfoliated teeth; human primary second molars-with coronal dentin acute caries and a root resorption lower than two-thirds, and without developmental defects, restoration, plaque, cracking and discoloration were used. Teeth having D3, caries limited to the outer half of the dentin were selected. ${ }^{8}$ A written consent was obtained from the adults responsible for the children that donated the teeth. Immediately after extraction teeth were stored in saline for a maximum of 1 week prior to meticulous cleaning of the root surface from debris and residual soft tissue. Specimens were intermediately stored at $-27^{\circ} \mathrm{C}$; surfaces were wetted with saline prior to freezing but no other liquids were added.

\section{Specimen preparation}

Enamel were removed by grinding the sample teeth perpendicularly to the carious surface using a slow-speed diamond saw under continuous water cooling until a flat surface was formed and the lesion in dentin was exposed.

\section{Caries removal and evaluation}

The teeth were randomly divided into three experimental groups, each of which containing ten teeth. In Group 1, caries was mechanically removed with a carbon-steel round bur IISO No. 012, Claudius Ash Ltd., Potters Bar, Herts., UK) in a contra-angle speed- reducing hand piece (400rpm). The maximum cutting dimension of the bur was $1.21 \mathrm{~mm}$. In Group 2, ten cavities were prepared using hand excavation with a straight spoon excavator (Ash G5, Claudius Ash Ltd., Potters Bar, Herts., UKJ from the occlusal aspect of the lesion. In Group 3, Carisolv Gel Multimix (MediTeam Dental AB, Gothenburg, Sweden) was used for the process of caries removal. Carisolv consists of two carboxymethycellulose-based gels:

1. A red gel containing $0.1 \mathrm{M}$ amino acids (glutamic acid, leucine lysine), $\mathrm{NaCl}, \mathrm{NaOH}$, erythrosine and purified water.

2. A clear liquid of sodium hypochlorite ( $\mathrm{NaOCl}$ $0.5 \%(\mathrm{w} / \mathrm{v})$

The two separate gels, which had been stored at $4^{\circ} \mathrm{C}$ before use, were allowed to regain room temperature over a $12 \mathrm{~h}$ period prior to use. The dentin caries was first covered with the pink Carisolv gel. After $30 \mathrm{~s}$, the carious dentin was gently scraped with hand instruments to remove softened carious tissue. A special hand instrument (Carisolv instruments 2, 3 and 4) fit for the dimension and availability of the cavity was chosen and the softened carious dentin on the surface was scraped. These instruments have a 90-degree edge as opposed to a sharp cutting profile. When the gel becomes heavily contaminated with debris, it was removed with cotton pellets and more fresh gel was applied. The procedure was repeated until the gel no longer became cloudy and all surfaces of the cavity were hard on probing, indicating that no soft dentin caries was left. After complete caries removal, the remaining gel was removed with wet and dry cotton pellets.

In groups 1 and 2, a caries detector dye (Caries Detector, Kuraray Medical Inc., Tokyo, Japan) contain $1 \%$ acid red in propylene glycol was used according to the manufacturer's instructions for reduces the risk of removing sound dentin. After repeating Caries Detector staining, removal of carious dentin was stopped when the cavity floor dentin stained light pink in groups. The staining conditions of caries detector were judged by 3 investigators.

The process of caries removal in all groups has been carried out by the same examiner (T.G.). Upon completion of the excavating procedures, all specimens were rinsed with an air-water spray for $1 \mathrm{~min}$.

\section{Examinations}

A blinded examiner (Y.Y.) checked all samples for residual caries using a visual-tactile examination, a laser fluorescence device and histological examination. The examinations were carried out as follows:

Visual and tactile evaluation

A calibration was carried out prior to the visual and tactile examination. The experienced examiner 
made a decision on the presence or absence of residual caries according to the hardness of the marked lesion area. For the visual-tactile examination, the teeth were dried briefly using compressed air and were viewed under a dental unit light. The visual criteria included the absence of any discoloration. The tactile criteria included the smooth passage of hard to a dental probe and absence of a catch or a "tug-back" sensation. The efficacy of caries removal was graded as complete or incomplete and numerically scored 0 or 1.

\section{Laser fluorescence examination}

The measurements were made for each sample using DIAGNOdent. After confirmation of accurate unit setting, the device was first calibrated using a ceramic plate according to the manufacturer's instructions (KaVo, Biberach, Germanyl. The fluorescence of a sound spot on the smooth surface of the tooth was measured in order to provide a baseline reading for each tooth (secondary calibration) and again after every $10^{\text {th }}$ tooth. With tip A attached to the apparatus, DIAGNOdent values were carefully measured with the apex of the tip in contact with the surface of the carious dentin of the cavity floor. The samples were dried briefly using compressed air. The DIAGNOdent readings taken prior to caries removal and was provided a baseline value for each tooth. After removal of the caries-infected dentin DIAGNOdent readings were retaken in all groups. The highest DIAGNOdent reading from the marked lesion area was recorded for the each sample. A blinded examiner evaluated differences among initial and final DIAGNOdent readings within the same groups. Three measurements in each surface were taken and the mean value was calculated. The cutoff for sound tissue was set at 15. ${ }^{10}$

\section{Histological examination}

Prior to the histological examination, color photographs of the caries removal surfaces were taken to assist the subsequent histological examination. The specimens' roots were separated from the coronal part and each tooth was prepared for histological examination. Teeth were hemi-sectioned in a mesial-distal direction through the center of the marked lesion area with a high-speed drill and fine diamond bur. Speed was set at $800 \mathrm{rpm}$ and a moderate weight $(100$ g) was chosen to guide the diamond-coated saw blade during the cutting procedure. One half of each tooth was processed for histological evaluations. Subsequently, specimens were dried in alcohol solvents of increasing concentration and embedded in Technovit 9100 New (Heraeus Kulzer, Wehrheim, Germany). Serial sections of $200 \mu \mathrm{m}$ were prepared and polished to a final thickness of approximately $150 \mu \mathrm{m}$. Wet sections were viewed under a polarized light microscope (Axiovert 135, Carl Zeiss, Jena, Germany) at x10 magnification. Specimens were immersed in water, and the test sites were viewed under a polarized light microscope for the presence or absence of residual caries. ${ }^{15}$ To prevent examiner bias, the reproducibility of the histological validation was determined by re-examining the sections of teeth after a minimum break of 1 week. The examiner was blind to the previous results. Intra-examiner correlation (Pearson) was $r_{p}=0.91-0.98$.

\section{SEM evaluation}

One half of each tooth was prepared for scanning electron microscope (SEM) investigation of the treated dentinal surface morphology. The samples were rinsed and were ultrasonically cleaned during 12 minutes. Then, the specimens were mounted separately on aluminum stubs, coated with gold/palladium by means of an ion coating equipment (SEM Coating Unit E 500, POLARON Equipment Limited, Barcelona, Spain), then evaluated under SEM (JSM-6400, JEOL, Tokyo, Japan) and micrographs were obtained.

\section{Statistical analysis}

Statistical analysis were performed by means of SPSS 13.0 for Windows software ISPSS Inc., Chicago IL, USA). Cohen's Kappa was calculated for intra-examiner calibration of detecting residual dentinal caries. The Kappa values were determined using the established cut-off levels for caries status. A two-way ANOVA with repeated measures were applied to do the comparisons among the groups and between the initial and final DIAGNOdent measures. In each group, whether there is a difference between methods used for determining residual caries is analyzed by McNemar test, and differences in the comparisons between all the groups are analyzed by Fischer's 
exact test. Statistical significance were set at $\mathrm{P}=.05$.

\section{RESULTS}

Our initial Cohen's Kappa evaluation of intraexaminer reproducibility agreement produced Kappa values from 0.49 to 0.93 .

Therewasnot differenceamong the groupsin the initial DIAGNOdent readings. There were statistical differences among initial and final DIAGNOdent values within the same groups $(\mathrm{P}<.05)$ (Table 1). In addition, the mean DIAGNOdent readings between all groups were stated significant $(P<05)$ and the mean DIAGNOdent values in the groups 2 and 3 were significantly higher than those obtained from the group 1 (Table 1).

In group 1, the caries were observed to be completely removed when inspecting the lesion region by the visual-tactile examination and DIAGNOdent method. There was an agreement between each method and histological gold standard ( $P>$.05).

After the inspection of the lesion region in group 2, caries in 4 teeth by the visual-tactile examination and was observed, while caries in 7 teeth was determined by using the DIAGNOdent
(Table 2). Caries determination done by two methods has been found to be common for 4 teeth in group 2. In both groups, no significant difference has been observed between the visualtactile examination-DIAGNOdent by the McNemar analysis of the data obtained ( $P>$.05) (Table 2). The histological examination of 10 teeth revealed that 5 teeth had residual caries.

In the assessment of the caries removed surfaces, in group 3, residual caries in only 1 tooth in the visual-tactile examination and residual caries in 4 teeth in the case using DIAGNOdent, examination were detected (Table 2). The detection by visual-tactile and DIAGNOdent methods has been found to be common for 1 tooth in group 3 . In the analysis of the data in group 3, no statistically significant difference among two methods was observed ( $P>$.05). In case of using histological examination, the caries was detected in 2 teeth.

In the analysis of the data revealed significant differences among the groups in terms of residual caries detection $(P<.05)$.

SEM images of the surface morphology are shown in Figures 1-3. In group 1, dentin surfaces with a smooth and homogenous smear layer are observed in Figure 1. The surfaces appeared

Table 1. DIAGNOdent values before treatment and after removal of the carious dentin.

\begin{tabular}{lcccc}
\hline \multirow{2}{*}{ Groups } & N & \multicolumn{2}{c}{ DIAGNOdent Values ( \pm SD $)$} & \multirow{2}{*}{ Sig } \\
\cline { 3 - 4 } & & Initial & Final & $*$ \\
\hline Group 1 & 10 & $54.4 \pm 17.4^{\mathrm{a}}$ & $9.2 \pm 5.3^{\mathrm{a}}$ & $*$ \\
Group 2 & 10 & $60.7 \pm 13.2^{\mathrm{a}}$ & $25.8 \pm 15.2^{\mathrm{b}}$ & $*$ \\
Group 3 & 10 & $56.4 \pm 12.2^{\mathrm{a}}$ & $20.1 \pm 11.8^{\mathrm{c}}$ & $*$ \\
\hline
\end{tabular}

*: $P<.05$, If marked by the same letter, the difference between the groups is statistically insignificant ( $>$ >05).

Table 2. Distribution of caries evaluation results for each method.

\begin{tabular}{|c|c|c|c|c|}
\hline & \multicolumn{4}{|c|}{ Caries detection methods $(n=30)$} \\
\hline & $\begin{array}{l}\text { Visual tactile } \\
\text { examination }\end{array}$ & $\begin{array}{c}\text { DIAGNOdent } \\
\text { measurements }\end{array}$ & $\begin{array}{c}\text { Histological } \\
\text { evaluation }\end{array}$ & \\
\hline Caries removal methods & $\begin{array}{c}\text { Caries } \\
\text { Present/Absent }\end{array}$ & $\begin{array}{c}\text { Caries } \\
\text { Present/Absent }\end{array}$ & $\begin{array}{c}\text { Caries } \\
\text { Present/Absent }\end{array}$ & $P$ value \\
\hline Bur excavation & $0 / 10$ & $0 / 10$ & $0 / 10$ & 1.000 \\
\hline Hand excavation & $4 / 6$ & $7 / 3$ & $5 / 5$ & 0.250 \\
\hline Carisolv excavation & $1 / 9$ & $4 / 6$ & $2 / 8$ & 0.250 \\
\hline Total & $5 / 25$ & $11 / 19$ & $7 / 23$ & $0.031 *$ \\
\hline
\end{tabular}

$* P<.05$ 
rougher with irregular particles scattered throughout the surface area. The smearing effect was accentuated and the dentin had apparently been removed in layers as opposed to being irregularly chipped away.

Figure 2 shows that dentin surfaces in group 2 are very rough and tubule openings are occluded with a smear layer. The surface finish of handexcavated cavity was described as having a grated effect. This effect was accentuated with some evidence of tubule openings. It appeared that the openings had been occluded to a significant degree (Figure 2).

Finally, rough dentin surfaces, open dentinal tubule orifice and no smear layer are observed in group 3, as illustrated in Figure 3. The flaky effect was accentuated due to the lack of an obvious smear layer. Even, there was evidence of tubule openings (Figure 3).

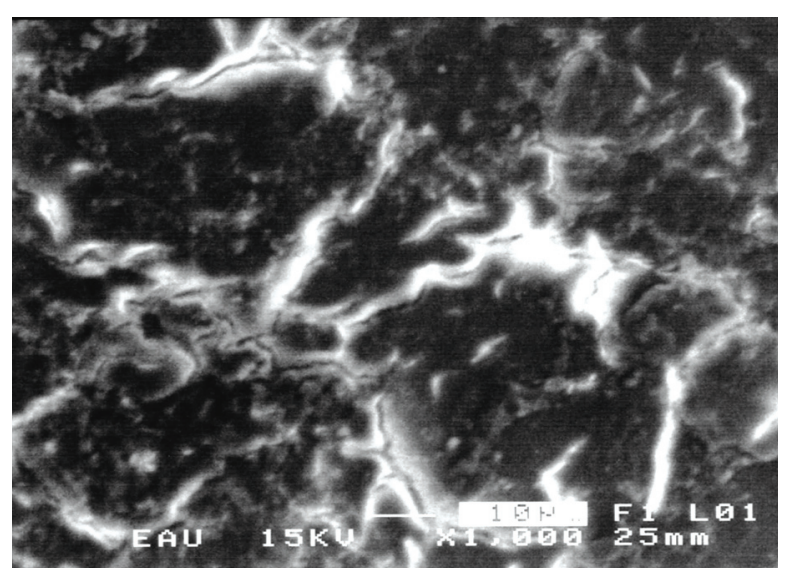

Figure 1. Scanning electron micrograph of the bur-prepared cavity with evidence of smearing and tubule orizfice occlusion (original magnification at $X 1000$ ).

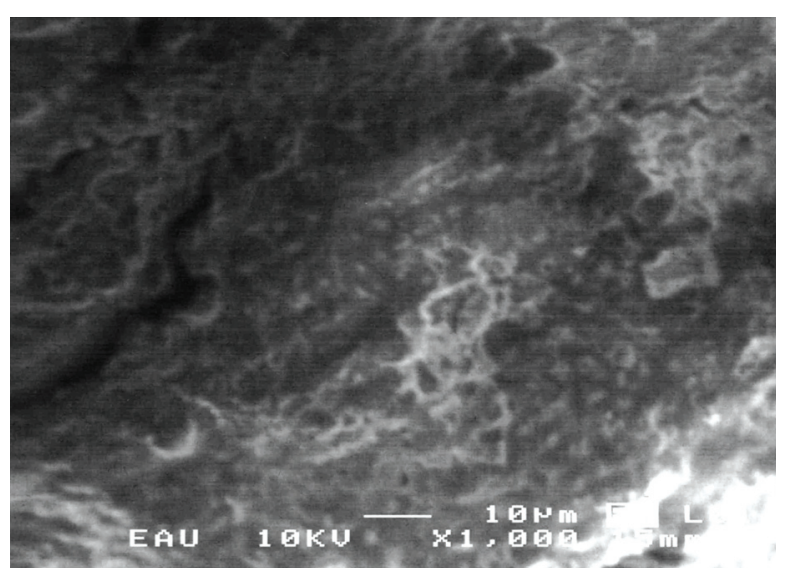

Figure 2. Scanning electron micrograph of the excavatorprepared dentin showing the very rough surface finish with smearing (original magnification at $X 1000$ ).

\section{DISCUSSION}

In carious dentin removal with Caries Detector, when the outer layer of carious dentin is stained red, it must be removed, and when the inner layer of carious dentin is stained light pink, it must be preserved. ${ }^{6}$ In the present study, carious dentin was detected using a Caries Detector dye in groups 1 and 2. Several approaches for the selective removal of infected dentine have been introduced during the last decades including the use of Caries Detector dyes and chemomechanical caries removal technique.

Hossain et $\mathrm{al}^{14}$ recommended a combination of hardness testing by an explorer and DIAGNOdent. The usefulness of DIAGNOdent device for the assessment of carious dentin removal has previously been reported by Lussi et al. ${ }^{16}$ The distribution of DIAGNOdent values in primary teeth exhibits a difference in an unimportant degree with respect to that of permanent teeth. ${ }^{17}$ The best performance of DIAGNOdent is said to be in dentinal caries which is emerged by opening the fissure ${ }^{18}$ and to predict the extension of caries lesions, mainly at dentinal caries threshold in primary teeth. ${ }^{19}$

Using histopathological and microhardness evaluation, Mendes et al $^{19}$ showed that the best DIAGNOdent performance was obtained at the $D 3$, caries limited to the outer half of the dentin threshold. It has been shown that the DIAGNOdent readings were useful to facilitate accurate removal of the caries-infected dentin. ${ }^{20}$ Further, Yazici et al ${ }^{9}$ stated that DIAGNOdent agreed better than the caries detector dye with the histological evaluation in assessing teeth with residual caries.

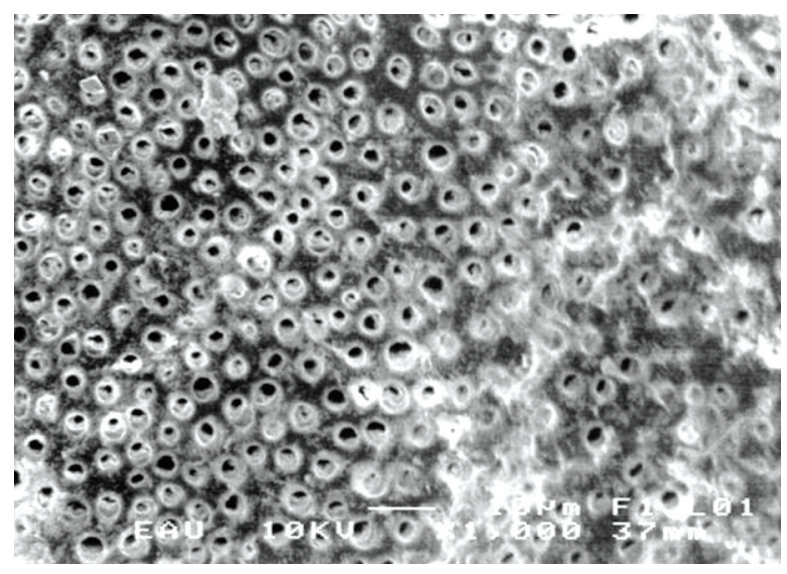

Figure 3. Scanning electron micrograph showing cavity surface prepared using Carisolv gel showing of the open tubular orifices as well as microcracks on the surface loriginal magnification at $X 1000)$. 
Thus, in the present study; we used DIAGNOdent readings to observe the condition of the dentin after removal of the caries-infected dentin tissues that were stained with caries detecting dye.

It has been documented DIAGNOdent values are influenced by the amount of collagen and the diameter of apatite crystals in human dentin. ${ }^{21}$ According to Hosoya et $\mathrm{al}^{22}$ the accuracy of DIAGNOdent readings to detect completeness of dentin caries excavation is lower in the primary teeth than in the permanent teeth. Thus, for the primary teeth, it may be necessary to use both a caries detecting dye and the DIAGNOdent device to assess the extent of caries removal. ${ }^{23}$ Krause et $\mathrm{al}^{24}$ reported that approaching in the dental pulp, increasing fluorescence values might lead to unnecessary pulp exposures.

In this study, DIAGNOdent evaluation methods used to confirm that the carious dentin is completely removed. However, the Carisolv do not completely remove remineralizable dentin; therefore, this method may give misleading results to operators. We discount DIAGNOdent values that can be used as a guide for removing the carious dentin outer layer with the Carisolv excavation method. Yamada et $\mathrm{al}^{25}$ stated that the efficiency of complete carious dentin removal by the Carisolv system in no longer difficult when a proper clinical guide le.g. DIAGNOdent assessment and caries detector solution) is used. The laser fluorescence readings reflect changes in the organic material rather than in the inorganic content of the teeth. ${ }^{26}$

The fluorescent molecules responsible for the increase of fluorescence in carious tissue seem to be porphyrins, mainly proto-porphyrin IX. These porphyrins could be synthesized by several microorganisms in carious lesions. ${ }^{26}$ When these molecules were mixed with synthetic hydroxyapatite or dried on tooth surfaces, there was a marked increase in the DIAGNOdent readings. ${ }^{26}$ In the present study, since Carisolv gel contains sodium hypochlorite, this fact could be the reason to the negative performance of the DIAGNOdent in detecting residual dentin caries after removal with the Carisolv.

In this study, it has been observed that dentine surfaces are covered with a smear layer after the use of bur in the caries removal while they have roughness, irregularity, undercut and covered with a smear layer in the hand excavation. However, the cutting action was not as vigorous as that of a bur. Additionally, in the group with caries removal with Carisolv system, the two factors (mechanical preparation technique and high $\mathrm{pH}$ ) will probably result in a minimal smear layer being formed, thus the tubule orifices appeared patent in every sample when using the gel. There also seemed to be evidence of a greater of cracking in the dentin surface. These micro cracks might have been the result of the hydrophilic nature of the gel causing dehydration of the dentin surface. ${ }^{27}$ The cavity prepared by using the Carisolv gel showed a generalized flaky and roughened surface.

Hosoya et al ${ }^{22}$ reported that Carisolv treatment removed the smear layer and opened dentinal tubules of primary dentin and Carisolv may have dissolved more collagen tissue in the primary dentin than in the permanent dentin, perhaps because the lower mineral content offers less protection against the non-specific deproteinizing effects of $\mathrm{NaOCl}$. According to Hossain et al, ${ }^{14}$ the dentinal surfaces after Carisolv treatment were irregular, and there remained a minimal debrislike smear layer and most of the dentinal tubules were opened. These results were consistent with the present study's findings.

Peric and Markovic ${ }^{28}$ was judged that caries status of dentine according to clinical criteria (probing and visual inspection) after caries removed using conventional rotary instruments and Carisolv. These authors found that all examined dentine surfaces were clinically caries free in histological evaluation. Upon comparison, our results were similar to the results of these researchers: no significant difference between the two groups was found in residual caries detection using visual tactile examination and histological evaluation.

In a study, Flückiger et a ${ }^{29}$ showed that there was no significant difference in the amount of residual caries in the cavity walls treated using the Carisolv and conventional hand excavation in the histological analysis. The findings by the researchers show harmony with the results of our study. However, in the present study DIAGNOdent readings and histological evaluations were generally high in hand excavation group comparison with the other groups. The reason for the higher readings the results of evaluation by the DIAGNOdent are not affected by the surface structure of the dentin, for 
example, the micro cracks (Group 2), the smear layer and the orifices of the dentinal tubules. The hypothesis was therefore accepted. In addition, the DIAGNOdent values were much influenced by the internal structure of the evaluated dentin when the thickness of the layer of sound dentin between the tip of the DIAGNOdent and the internal structure was less than $0.2-0.3 \mathrm{~mm} \cdot{ }^{30}$

\section{CONCLUSIONS}

The present study was performed under in vitro conditions. The use of DIAGNOdent in detecting residual caries requires further studies under in vivo conditions. Based on histological and scanning electron microscope analyses it could be concluded that DIAGNOdent and visual tactile examination can detect the remaining dentin as sound after bur excavation. However, DIAGNOdent abilities to detect residual caries were low after hand excavation and chemomechanical caries removal and, therefore, it is advisable to test the residual caries with an additional diagnostic tool such as visual tactile examination.

\section{REFERENCES}

1. Banerjee A, Watson TF, Kidd EA. Dentine caries excavation: A review of current clinical techniques. $\mathrm{Br}$ Dent $J$ $2000 ; 188: 476-482$.

2. Kuboki Y, Liu CF, Fusayama T. Mechanism of differential staining in carious dentin. J Dent Res 1983;62:713-714.

3. Fusayama T, Okuse K, Hosoda H. Relationship between hardness, discoloration, and microbial invasion in carious dentin. J Dent Res 1966;45:1033-1046.

4. Van de Rijke JW. Use of dyes in cariology. Int Dent $J$ 1991;41:111-116.

5. Yip HK, Stevenson AG, Beeley JA. The specificity of caries detector dyes in cavity preparation. Br Dent J1994;176:417421.

6. Lussi A, Megert B, Longbottom C, Reich E, Francescut P. Clinical performance of a laser fluorescence device for detection of occlusal caries lesions. Eur J Oral Sci 2001;109:14-19.

7. Iwami Y, Shimizu A, Hayashi M, Takeshige F, Ebisu S. Relationship between colors of carious dentin and laser fluorescence evaluations in caries diagnosis. Dent Mater $J$ 2006;25:584-590.

8. Mendes FM, Hissadomi M, Imparato JC. Effects of drying time and the presence of plaque on the in vitro performance of laser fluorescence in occlusal caries of primary teeth. Caries Res 2004;38:104-148.
9. Yazici AR, Baseren M, Gokalp S. The in vitro performance of laser fluorescence and caries-detector dye for detecting residual carious dentin during tooth preparation. Quintessence Int 2005;36:417-422.

10. Lennon AM, Buchalla W, Switalski L, Stookey GK. Residual caries detection using visible fluorescence. Caries Res 2002;36:315-319

11. Lozano-Chourio MA, Zambrano O, González H, Quero M. Clinical randomized controlled trial of chemomechanical caries removal (Carisolv). Int J Paediatr Dent 2006;16:161167.

12. Splieth C, Rosin M, Gellissen B. Determination of residual dentine caries after conventional mechanical and chemomechanical caries removal with Carisolv. Clin Oral Invest 2001;5:250-253.

13. Yazici AR, Atilla P, Ozgunaltay G, Muftuoglu S. In vitro comparison of the efficacy of Carisolv and conventional rotary instrument in caries removal. $J$ Oral Rehabil 2003;30:1177-1172.

14. Hossain M, Nakamura Y, Tamaki Y, Yamada Y, Jayavardena JA, Matsumoto K. Dentinal composition and knoop hardness measurements of cavity floor following carious dentin removal with Carisolv. Oper Dent 2003;28:346-341.

15. König K, Flemming G, Hibst R. Laser-induced autofluorescence spectroscopy of dental caries. Cell Mol Biol 1998;44:1293-1300.

16. Lussi A, Hibst R, Paulus R. DIAGNOdent: an optical method for caries detection. J Dent Res 2004;83:80-83.

17. Lussi A, Francescut P. Performance of conventional and new methods for the detection of occlusal caries in deciduous teeth. Caries Res 2003;37:2-7.

18. Anttonen V, Seppä L, Hausen H. Clinical study of the use of the laser fluorescence device DIAGNOdent for detection of occlusal caries in children. Caries Res 2003;37:17-23.

19. Mendes FM, Siqueira WL, Mazzitelli JF, Pinheiro SL, Bengtson AL. Performance of DIAGNOdent ${ }^{\circledR}$ for detection and quantification of smooth-surface caries in primary teeth. J Dent 2005;33:79-74.

20. Iwami Y, Shimizu A, Narimatsu M, Hayashi M, Takeshige F, Ebisu S. Relationship between bacterial infection and evaluation using a laser fluorescence device, DIAGNOdent. Eur J Oral Sci 2004;112:419-423.

21. Suzaki A, Takahashi K, Sakuma K, Arimoto N, Senda A, Hayakava T. A fundamental study on clinical application of DIAGNOdent -Effects of demineralization and collagenase treatment of bovine dentin on the defective response of DIAGNOdent ${ }^{\text {TM }}$. Jpn J Conser Dent 2000;43:200-207.

22. Hosoya Y, Kawashita Y, Marshall GW, Goto G. Influence of CarisolvTM for resin adhesion to sound human primary dentin and young permanent dentin. J Dent 2001;29:163-171. 
23. Hosoya Y, Taguchi T, Tay FR. Evaluation of a new caries detecting dye for primary and permanent carious dentin. $J$ Dent 2007;35:137-143.

24. Krause F, Braun A, Eberhard J, Jepsen J. Laser fluorescence measurements compared to electrical resistance of residual dentine in excavated cavities in vivo. Caries Res 2007;41:135-140.

25. Yamada Y, Kimura Y, Hossain M, Kinoshita J, Shimizu Y, Matsumoto K. Caries removal with Carisolv system: Criteria evaluation and microleakage test. J Clin Pediatr Dent 2005;30:121-126.

26. Hibst R, Paulus R, Lussi A. Detection of occlusal caries by laser fluorescence: basic and clinical investigations. Med Laser Appl 2001;16:205-213.

27. Banerjee A, Kidd EAM, Watson TF. Scanning electron microscopic observations of human dentine after mechanical caries excavation. J Dent 2000;28:179-186.

28. Peric T, Markovic D. In vitro effectiveness of a chemomechanical method for caries removal. Eur J Paediatr Dent 2007;8:61-67.

29. Flückiger L, Waltimo T, Stich H, Lussi A. Comparison of chemomechanical caries removal using Carisolv ${ }^{\top M}$ or conventional hand excavation in deciduous teeth in vitro. $J$ Dent 2005;33:87-90.

30. Iwami Y, Shimizu A, Yamamoto H, Hayashi M, Takeshige F, Ebisu S. In vitro study of caries detection through sound dentin using a laser fluorescence device, DIAGNOdent. Eur J Oral Sci 2003;111:7-11. 